\title{
Carbon Nanomaterials-Promising Solid Lubricants to Tailor Friction and Wear
}

\author{
Sebastian Suarez ${ }^{1, *(1)}$ and Andreas Rosenkranz ${ }^{2, *(D)}$ \\ 1 Functional Materials, Saarland University, 66123 Saarbrücken, Germany \\ 2 Department for Chemical Engineering, Biotechnology and Materials, University of Chile, \\ Santiago 8320198, Chile \\ * Correspondence: s.suarez@mx.uni-saarland.de (S.S.); arosenkranz@ing.uchile.cl (A.R.)
}

Received: 14 June 2019; Accepted: 14 June 2019; Published: 18 June 2019

It is our pleasure to launch this Special Issue related to the application of carbon nanomaterials as solid lubricants to tailor friction and wear. In times of a continuously increasing energy demand, diminishing material resources, and more consciousness about global warming, the addressed topic has not only scientific but also significant economical and societal implications. Friction- and wear-related energy losses, which directly lead to increased operational costs, can account for up to several points of the GDP of an industrialized country [1]. Nowadays, the usage of oils enriched with certain additives such as phosphorus, sulphur, and zinc is seen as the easiest way to efficiently reduce friction and wear. However, due to environmental risks brought by these elements, their incorporation to lubricants is becoming more and more restricted, which makes the need for alternative and more sustainable ways/approaches to reduce friction and wear stronger than ever before.

In this regard, the usage of solid lubricants can be considered as a promising alternative, which finds its application niche in engineering fields where environmental conditions or the load collective do not allow one to use of liquid lubricants. In this regard, certain mechanical systems in which the operation temperature exceeds the degradation temperature of the oil, or for which the applied pressures would unavoidably result in a metal-metal contact, lie within this. Other important application fields may not have the widespread occurrence of the previously mentioned examples, but are also highly relevant. The best example is friction in spacecraft applications, for which all solutions are unique and must properly respond to extreme environments (e.g., vacuum and radiation).

The family of materials used for solid lubrication is rather broad and includes soft metals, transition metal dichalcogenides, polymers, and carbon-based materials, among others [2]. In this context, carbon-based solid lubricants have been gaining remarkable attention in the scientific community lately, despite having been used for a long time already (especially graphite). The lubrication principle of graphite is supported by the low shear strength between its constituent graphene layers, hence allowing for the delamination of graphite flakes. However, this happens only under certain environmental conditions, for which humidity plays a fundamental role.

In recent years, the rise of other graphitic carbon allotropes has opened new research paths in the field of tribology, since these new carbon structures can function in a wider range of conditions. Furthermore, their different morphologies may induce different lubricant mechanisms, thus tailoring friction and wear in a more efficient way. This increases not only their applicability range but also their ability to provide a tailored solution for each particular case. These materials should ideally be subjected to different experimental conditions such as varying temperature, humidity, contact pressure, and material paring, in order to have a full behavioural description to effectively assign them and define parameters for their proper functioning. Consequently, a detailed description of the underlying friction and wear mechanisms of these new carbon allotropes is of utmost importance to guarantee their successful operation as solid lubricants. Additionally, undertaking a thorough investigation 
and attaining an understanding of tribologically-induced morphological, structural, and chemical changes of these carbon structures during sliding is, to our understanding, another key aspect to further optimize these new solid lubricants.

We hope that the contributions contained in this Special Issue provide the reader with a good overview of both the fundamental and applied characteristics of different carbon-based solid lubrication systems under diverse tribological conditions, further extending — or even kick-starting — research lines in this exciting field.

\section{References}

1. Holmberg, K.; Erdemir, A. Influence of tribology on global energy consumption, costs and emissions. Friction 2017, 5, 263-284. [CrossRef]

2. Scharf, T.; Prasad, S. Solid Lubricants: A Review. J. Mater. Sci. 2013, 48, 511-531. [CrossRef]

(C) 2019 by the authors. Licensee MDPI, Basel, Switzerland. This article is an open access article distributed under the terms and conditions of the Creative Commons Attribution (CC BY) license (http://creativecommons.org/licenses/by/4.0/). 